\title{
Analytical Model for Zones Distributions in Non-Horizontal Miscible WAG Injection
}

\author{
Mehran Namani ${ }^{1}$, Jon Kleppe ${ }^{1}$, Lars Høier ${ }^{1,2}$, Hassan Karimaie ${ }^{1,3}$ \& Ole Torsæter ${ }^{1}$ \\ ${ }^{1}$ Department of Petroleum Engineering and Applied Geophysics, Norwegian University of Science and \\ Technology, Trondheim, Norway \\ ${ }^{2}$ Statoil, Trondheim, Norway \\ ${ }^{3}$ Aker Solutions, Trondheim, Norway \\ Correspondence: Mehran Namani, Department of Petroleum Engineering and Applied Geophysics, Norwegian \\ University of Science and Technology, S. P. Andersens veg 15a, Trondheim 7491, Norway. Tel: 47-735-94949. \\ E-mail: mehran.namani@ntnu.no
}

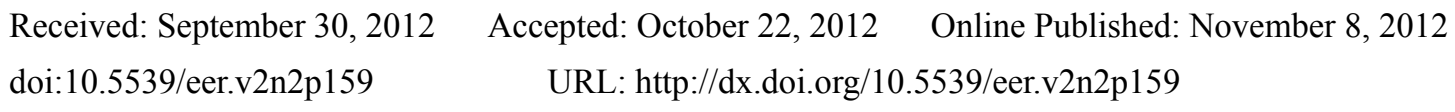

\begin{abstract}
The advantages of WAG (water-alternating-gas) injection compared to gas injection and waterflooding, from technical and economical points of view, are results of creating a mixed zone in which both water and gas are flowing simultaneously. Previously, an analytical model was developed in the literature to evaluate complete segregation distance and zone distributions for horizontal miscible WAG injection models. Even though this analytical model is derived using simplifying assumptions, it still gives good insight into the design of new projects when the lack of data does not allow a detailed full field reservoir simulation to be carried out. On the other hand, the advantages and disadvantages of applying WAG injection in dipped reservoirs, and up-dip or down-dip patterns are still in question while there isn't any analytical model available for analyzing process in this type of reservoir. This paper develops analytical equations for a non-horizontal model and investigates the effect of dip angle on segregation effects in miscible WAG injection process. Up-dip versus down-dip WAG injection is another subject which will be discussed in this paper.
\end{abstract}

Keywords: miscible WAG, non-horizontal model, segregation distance, zone distribution

\section{Introduction}

For several decades, enriched or high-pressure gas injection and also injection of miscible slug of liquefied petroleum gas (LPG) chased by lighter hydrocarbon gases as methane have been in the spotlight for enhanced oil recovery, as secondary and tertiary methods, due to their excellent displacement efficiency. Despite excellent displacement efficiency which can be even $100 \%$ in the swept area (Faisal, Bisdom, Zhumabek, Mojaddam, Zadeh, \& Rossen, 2009; Lake, 1989), poor sweep efficiency of gas injection due to viscous fingering, tonguing and segregation resulted by mobility and gravity effects impairs the recovery and economical advantages of such a process, especially in larger reservoirs with more complicated petrophysical characteristics. Aiming to increase sweep efficiency, different attempts have been carried out to explore new techniques in gas injection processes, including near-miscible and multi-contact miscible gas injection methods, which can improve sweep efficiency, as well as displacement efficiency; but still the sweep efficiency of field cases are not as promising as theoretical forecast.

Theoretically, or based on some one-dimensional flow experiments, the best microscopic efficiency in gas injection processes is supposed to be reached when the operating pressure is above minimum miscibility pressure (MMP). In the other words, for the most field scale projects, the gas that has been selected for the injection intended to be enriched beyond multi-contact miscibility. Sometimes, the enriching process is continued even up to or above first-contact miscibility to make sure that miscibility will not be lost during multiple contacts and dispersive mixing. Field scale simulations by Pande (1992) showed that this approach which is mostly concluded from one-dimensional flow experiments cannot always be optimum point. Namely, hydrocarbon gases enriched up to or less than necessary composition for a multi-contact miscibility can exhibit larger driving forces both in gravity and viscous cross flow which as a result can favor both displacement and sweep efficiency in 
heterogeneous reservoirs. For instance, hydrocarbon gas injection simulation both as secondary and tertiary methods showed that enrichment at or below multi-contact miscibility can yield as well or even better results than injection with more enriched gases.

Thomas, Holowach, Zhou, Bennion, and Bennion (1994) concluded from simulation studies that good recoveries can be achieved by low-IFT gas injection, and in the most cases, zero-IFT conditions, which expresses a miscible process is not necessary. Using a high-pressure glass micromodel, Sohrabi, Danesh, Tehrani and Jamiolahmady (2008) also confirmed that near-miscible gas injection gives good recoveries both as a secondary recovery technique and in waterflooded of reservoirs. These contradictory results happen mostly because of poor gas sweep efficiency and there are more examples of this mechanism explained in the literature. Caudle and Dyes (1958) and Dyes, Caudle and Erickson (1954) have discussed this mechanism in miscible process and in a pattern injection system, compared to waterflooding and also, the effect of mobility ratio on oil production after breakthrough.

Based on this background, the injection of water together with miscible gas was proposed and implemented to reduce the relative permeability and effective mobility of gas phase, and consequently, creating less fingering and/or tonguing of gas, effectively; but later, considering reduced injectivity of simultaneous water and gas injection and for feasibility issues in field cases, WAG (water-alternating-gas) injection has been introduced as an alternative and of course, an improve to the gas injection process (Stone, 1982; Caudle \& Dyes, 1958). Economical advantage of such a method regarding less gas demand is also another point makes it more attractive in field scale.

Although implementing this method improves both recovery and economical conditions considerably, early gravity segregation of injected phases, particularly for gas, decreases the expected ideal recoveries (Stone, 1982; Jamshidnezhad, Shen, Kool, Mojaddam Zadeh, \& Rossen, 2010; Blackwell, Terry, Rayne, Lindley, \& Henderson, 1960). Gravity segregation is basically a slow process and needs time to be fulfilled. So, considering the higher viscous forces close to the injection well and the time which is required for gravity segregation, a good sweep takes place close to injection well but it deteriorates as injectants are getting far from injection perforations. Finally at a certain distance from injection well, complete segregation of injected phases happens and three-phase flow will not take place anymore (Figure 1). There are several reservoir and operating parameters affecting this distance which will be called "complete segregation distance" from now on. Stone (1982) believes that injection rate, vertical permeability and density difference between water and gas are the main parameters affecting this distance. He used analytical equations to predict complete segregation distance and other boundaries for this process in a homogeneous horizontal reservoir, and compared the prediction with reservoir simulation results. In this study we will derive equations for a non-horizontal reservoir based on Stone's model (1982) and Jenkins' developments (1984), and will compare results with a horizontal model to discuss the advantages and disadvantages of WAG injection in a dipped reservoir.

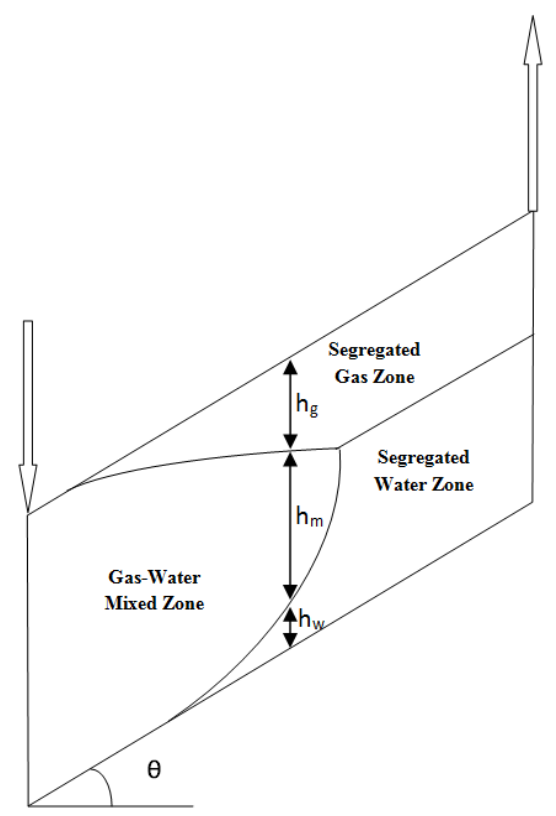

Figure 1. Steady state zone distribution in a non-horizontal WAG injection model 


\section{Problem Description, Assumptions and Method}

As explained, WAG injection has been implemented as an alternative to a gas injection process to increase the sweep efficiency, keeping the displacement efficiency constant or even improving it. Stone (1982) developed an analytical model to analyze the macroscopic flow regions. He assumed the process to be analyzed at steady state conditions, where different flow zones including a gas-water mixed zone and segregated gas and water zones, are fixed. This model is very helpful for pre-simulation design of a field scale WAG process. However, it is only applicable for horizontal models while in most of the reservoirs there is a dip angle between injection and production wells. In this paper this model is developed for a non-horizontal model (Figure 1), and theoretically, the situations for both up-dip and down-dip WAG injection are briefly discussed.

In this paper, in the order of getting a simplified analytical equation for the length of complete segregation we kept all of Stone's (1982) simplifying assumptions unchanged unless it is specifically mentioned otherwise. These assumptions and justifications have been discussed and completed in the literature in order to verify resulting equations (Rossen, van Duijn, Nguyen, Shen, \& Vikingstad, 2010). Meanwhile, we reviewed some of the most essential assumptions, here and during the derivation steps. After deriving related equations for complete segregation distance and boundaries of different zones presented in Figure 1, a set of seven non-linear equations with seven unknowns were obtained and solved by a procedure explained in the following.

Practically, the injection of water and gas is mostly implemented in alternative manner, because of the problems that arise by multiphase flow close to injection well and difficulties related to reduced injectivity. But still it may be assumed that we have a simultaneous injection of phases as long as the mixing of fluids near the wellbore is a valid assumption (Stone, 1982). This assumption is in particular correct if the WAG cycles are kept short. Faisal, et al. (2009) showed that a big improvement in WAG recovery happens when the conditions violate this assumption because of injectivity considerations which strongly depend on saturations adjacent to the wellbore. Increased injectivity lets one to inject with higher rates, i.e. higher viscous force, improved sweep and recovery.

\section{Results and Discussion}

The WAG ratio which is assumed to be constant along the reservoir model where a gas-water mixed zone exists, can be calculated by using Darcy's law for both phases:

$$
\text { WAG Ratio }=\frac{f_{w}}{f_{g}}=\left(\frac{k_{r w}}{\mu_{w}}\right)_{m}\left(\frac{\mu_{g}}{k_{r g}}\right)_{m}\left(\frac{\left.\left(\frac{d p}{d x}\right)\right)_{w}-\rho_{w} g \sin \theta}{\left(\frac{d p}{d x}\right) g-\rho_{g} g \sin \theta}\right)=\left(\frac{k_{r w}}{\mu_{w}}\right)_{m}\left(\frac{\mu_{g}}{k_{r g}}\right)_{m}\left(\frac{d \Phi_{w}}{d \Phi_{g}}\right)
$$

in which $f_{w}$ and $f_{g}$ are fractional flows of water and gas, respectively; $k_{r w}$ and $k_{r g}$ are relative permeabilities of water and gas, respectively, $\mu_{w}$ and $\mu_{g}$ are the viscosities of water and gas, respectively, $(d p / d x)_{w}$ and $(d p / d x)_{g}$ are pressure gradients along the length of water and gas phases, respectively, $\rho_{g}$ and $\rho_{w}$ are the densities of gas and water, respectively, g is gravity constant, $d \Phi_{w}$ and $d \Phi_{g}$ are flow potentials for water and gas, respectively, and finally, $\theta$ is the dip angle of the reservoir as shown in the Figure 1. Also, index $m$ denotes gas-water mixed zone.

Contrary to Stone's (1982) model for horizontal reservoirs, pressure gradients for two phases in non-horizontal models are not equal, but flow potentials for water and gas phases are equal. So, the Equation 1 will be simplified to

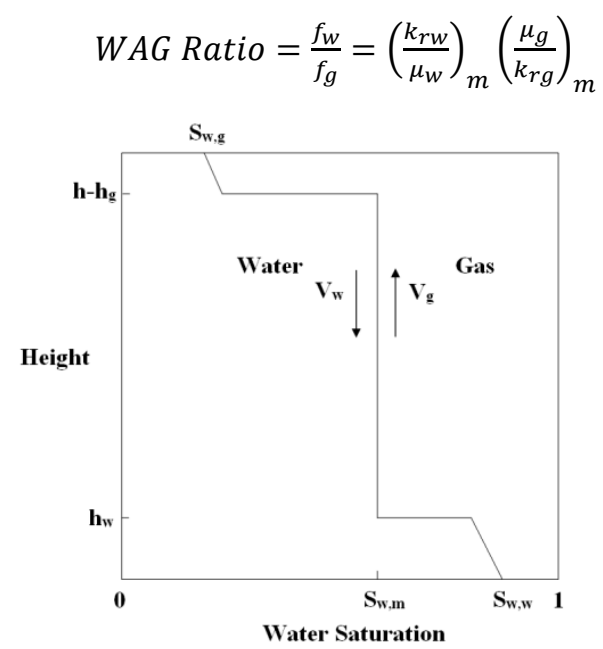

Figure 2. Vertical cross section of partially segregated zone (based on Stone, 1982) 
Figure 2 shows a vertical cross section of our model in the region in which partial segregation happened. Similar to the horizontal model of Stone (1982) evaluated using Buckley-Leverett theory (1941), there are three different water saturation values in this figure: water saturation in the gas-water mixed zone which is adjacent to the injection well and will be called "mixed zone" as abbreviation from now on, $S_{w, m}$, water saturation in the gas segregated zone, $S_{w, g}$, and water saturation in the water segregated zone, $S_{w, w}$. It is obvious that saturation distribution of an element in the mixed zone (Figures 1 and 2) will be similar to the horizontal model of Stone (1982) with a minor difference in the heights of different zones, $h_{m}, h_{w}$ and $h_{g}$, for up-dip or down-dip WAG injection, caused by accelerated gravity effect. As this cross sectional zone moves forward from injection toward production well, $h_{w}$ and $h_{g}$ get bigger and finally, at a particular distance from the injection well, these two meet. This distance is called the complete segregation distance.

Assuming that compressibility effects are negligible and by calculation of the segregation velocity in the mixed zone, we may write Darcy's law for countercurrent water and gas phases

$$
\begin{gathered}
v_{g}=-k_{v}\left(\frac{k_{r g}}{\mu_{g}}\right)_{m}\left[\frac{d p}{d z}+\rho_{g} g \sin \left(\frac{\pi}{2}-\theta\right)\right]=-k_{v}\left(\frac{k_{r g}}{\mu_{g}}\right)_{m}\left[\frac{d p}{d z}+\rho_{g} g \cos \theta\right] \\
v_{w}=-k_{v}\left(\frac{k_{r w}}{\mu_{w}}\right)_{m}\left[-\frac{d p}{d z}+\rho_{w} g \sin \left(-\frac{\pi}{2}-\theta\right)\right]=-k_{v}\left(\frac{k_{r w}}{\mu_{w}}\right)_{m}\left[-\frac{d p}{d z}-\rho_{w} g \cos \theta\right]
\end{gathered}
$$

Here $v_{g}$ and $v_{w}$ are gas and water segregation velocities in the mixed zone, $k_{v}$ is the vertical permeability of the homogeneous porous media and $d p / d z$ is the vertical pressure gradient along the segregation zone which can be assumed equal for both phases. Therefore, $d p / d z$ can be eliminated by replacing from Equation 4 into Equation 3

$$
v_{g}=-k_{v}\left(\frac{k_{r g}}{\mu_{g}}\right)_{m}\left[\left(\frac{v_{w}}{k_{v}}\right)\left(\frac{\mu_{w}}{k_{r w}}\right)_{m}-\rho_{w} g \cos (\theta)+\rho_{g} g \cos (\theta)\right]
$$

Assuming that the value of $v_{g}$ is equal to $v_{w}$, and rearranging and simplifying the resulting equation

$$
v=k_{v} \Delta \rho g \cos (\theta) \frac{\left(\frac{k_{r g}}{\mu_{g}}\right)_{m}}{\left[1+\left(\frac{k_{r g}}{\mu_{g}}\right)_{m}\left(\frac{\mu_{W}}{k_{r w}}\right)_{m}\right]}
$$

in which $v$ is the segregation velocity in the mixed zone and $\Delta \rho$ is the density difference between water and gas. Using Equation 2, we may reduce Equation 6 to

$$
v=\Delta \rho g \cos (\theta) k_{v} \frac{\left(\frac{f g}{f_{w}}\right)\left(\frac{k_{w} w}{\mu_{w}}\right)_{m}}{\left[1+\left(\frac{f g}{f_{w}}\right)\right]}
$$

or

$$
v=\Delta \rho g \cos (\theta) k_{v} \frac{f_{g}\left(\frac{k_{r w}}{\mu_{w}}\right)_{m}}{f_{w}+f_{g}}
$$

and considering

$$
f_{w}+f_{g}=1
$$

the final equation for the segregation velocity of a WAG process in a dipped reservoir will be resulted from Equation 8 as follow

$$
v=\Delta \rho g \cos (\theta) k_{v} f_{g}\left(\frac{k_{r w}}{\mu_{w}}\right)_{m}
$$

If we re-write Equation 2 as

$$
f_{w}\left(\frac{k_{r g}}{\mu_{g}}\right)=f_{g}\left(\frac{k_{r w}}{\mu_{w}}\right)
$$

the segregation velocity, Equation 10, can be alternatively re-written as

$$
v=\Delta \rho g \cos (\theta) k_{v} f_{w}\left(\frac{k_{r g}}{\mu_{g}}\right)_{m}
$$

This segregational velocity can be used in a material balance equation to calculate the length of complete segregation in a steady state WAG injection process for a non-horizontal model. Figure 3 shows a modified non-horizontal model based on the figure that Stone (1982) used to show the material balance equation implemented in a horizontal model. The material balance equation is

$$
q_{t} f_{w} f_{g}=v L_{G} w
$$


where $q_{t}$ is the total injection flow rate, $L_{G}$ is the complete segregation distance, and $\mathrm{w}$ is the width of the reservoir transverse to flow. This equation basically states that the primary assumptions of WAG for having both injectants completely mixed close to well and a uniform flow of these mixed phases along the whole gas-water mixed zone remain valid. As it is explained by Stone (1982), the flux of fluids right on both sides of the boundary of the segregated and mixed zones, where Equation 12 is written for, are similar but the type of fluids are different regarding the flow fractions, $f_{w}$ and $f_{g}$.

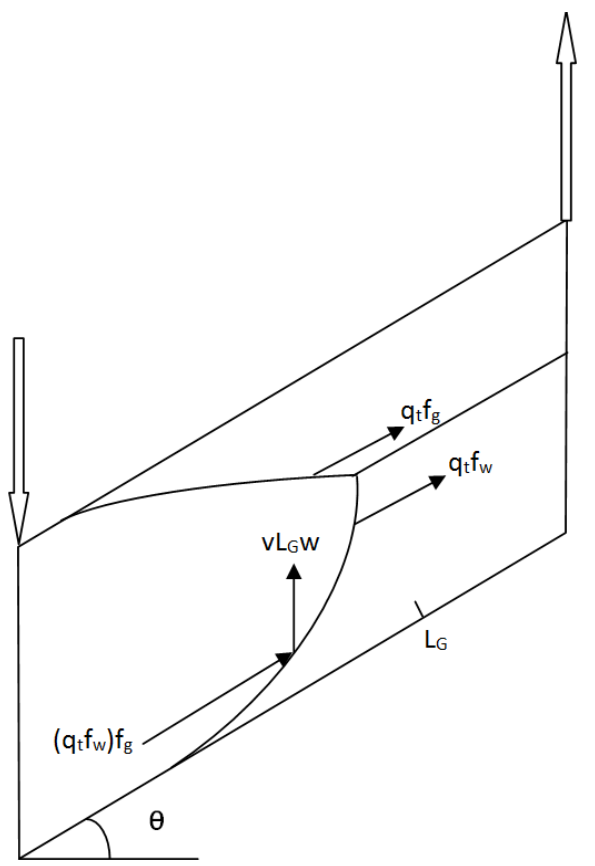

Figure 3. Steady state flow pattern in a non-horizontal WAG injection model

By replacing the segregational velocity of Equation 11 (or Equation 10) in the Equation 12 a much simpler equation will be obtained, which can be used for determining the complete segregation distance, $L_{G}$, in a dipped reservoir

$$
\begin{gathered}
q_{t} f_{w} f_{g}=\Delta \rho g \cos (\theta) k_{v} f_{g}\left(\frac{k_{r w}}{\mu_{w}}\right)_{m} L_{G} w \\
L_{G}=\left(\frac{q_{t}}{\Delta \rho g \cos (\theta) k_{v} w}\right)\left(\frac{f_{w}}{\left(\frac{k_{r w}}{\mu_{w}}\right)_{m}}\right)=\left(\frac{q_{t}}{\Delta \rho g \cos (\theta) k_{v} w}\right)\left(\frac{\frac{q_{w}}{q_{w}+q_{g}}}{\left(\frac{k_{r w}}{\mu_{w}}\right)_{m}}\right)
\end{gathered}
$$

in which $q_{w}$ and $q_{g}$ are water and gas injection rates, respectively, but may also represent segregated water and gas flow rates at the complete segregation distance, $\mathrm{L}_{\mathrm{G}}$, as well as water and gas flow rates in every other cross section inside this distance. Writing Darcy's law for the two and simplifying the terms, we obtain

$$
L_{G}=\left(\frac{q_{t}}{\Delta \rho g \cos (\theta) k_{v} w}\right)\left(\frac{1}{\left(\frac{k_{r w}}{\mu_{w}}\right)_{m}+\left(\frac{k_{r g}}{\mu_{g}}\right)_{m}}\right)
$$

Now that the complete segregation distance in a dipped model is determined, the next desired parameter is the boundaries of the mixed zone (Figure 1). Assuming similar flow potential in the flow direction for different regions, the Darcy's law for these regions may be written

$$
\begin{gathered}
\mathrm{q}_{\mathrm{w}}=-\left(\frac{\mathrm{k}_{\mathrm{rw}}}{\mu_{\mathrm{w}}}\right)_{\mathrm{w}}\left(\mathrm{k}_{\mathrm{h}} \mathrm{h}_{\mathrm{w}} \mathrm{w}\right)\left(\left(\frac{\Delta \mathrm{P}}{\Delta \mathrm{X}}\right)_{\mathrm{w}}+\rho_{\mathrm{w}} \mathrm{g} \sin (\theta)\right)=-\left(\frac{\mathrm{k}_{\mathrm{rw}}}{\mu_{\mathrm{w}}}\right)_{\mathrm{w}}\left(\mathrm{k}_{\mathrm{h}} \mathrm{h}_{\mathrm{w}} \mathrm{w}\right)\left(\mathrm{d} \Phi_{\mathrm{w}}\right) \\
\mathrm{q}_{\mathrm{g}}=-\left(\frac{\mathrm{k}_{\mathrm{rg}}}{\mu_{\mathrm{g}}}\right)_{\mathrm{g}}\left(\mathrm{k}_{\mathrm{h}} \mathrm{h}_{\mathrm{g}} \mathrm{w}\right)\left(\left(\frac{\Delta \mathrm{P}}{\Delta \mathrm{X}}\right)_{\mathrm{g}}+\rho_{\mathrm{g}} \mathrm{g} \sin (\theta)\right)=-\left(\frac{\mathrm{k}_{\mathrm{rg}}}{\mu_{\mathrm{g}}}\right)_{\mathrm{g}}\left(\mathrm{k}_{\mathrm{h}} \mathrm{h}_{\mathrm{g}} \mathrm{w}\right)\left(\mathrm{d} \Phi_{\mathrm{g}}\right)
\end{gathered}
$$




$$
\begin{aligned}
Q_{m}=-\left(\frac{k_{r w}}{\mu_{w}}\right)_{m}\left(k_{h} h_{m} w\right)\left(\left(\frac{\Delta P}{\Delta x}\right)_{m}+\rho_{\mathrm{w}} g \sin (\theta)\right)-\left(\frac{k_{r g}}{\mu_{g}}\right)_{m}\left(k_{h} h_{m} w\right)\left(\left(\frac{\Delta P}{\Delta x}\right)_{m}+\rho_{g} g \sin (\theta)\right)= \\
-\left(\frac{k_{r w}}{\mu_{w}}\right)_{m}\left(k_{h} h_{m} w\right)\left(d \Phi_{m}\right)-\left(\frac{k_{r g}}{\mu_{\mathrm{g}}}\right)_{m}\left(k_{h} h_{m} w\right)\left(d \Phi_{m}\right)
\end{aligned}
$$

Indices $\mathrm{w}, \mathrm{g}$ and $\mathrm{m}$ denote water segregated, gas segregated and mixed zones. All flow potentials in these equations are the same and supposed to be one of the unknowns we want to calculate. Having the flow potentials, the pressure gradient for each phase can be calculated. An important consideration must be expressed while replacing relative permeability values in these equations. Relative permeability in the Equation 16 has to be measured at the residual oil saturation of a steady state waterflood process while the relative permeability in Equation 17 has to be measured at the residual oil saturation of a steady state gas injection process. Also, the relative permeability values in the Equation 18 belong to a system in which a mixture of gas-water is injected to reach a steady-state residual saturation of oil. This typical saturation is shown as $S_{\mathrm{w}, \mathrm{m}}$ in the saturation profile shown in Figure 2. Jenkins (1984) explained a very useful theoretical approach, base on Buckley-Leverett theory (1941) to calculate these relative permeabilities.

To solve the above equations to get the boundaries of different regions, some auxiliary equations will be needed. Below supplementary equations by Stone (1982) can be used in this case:

$$
\begin{gathered}
q_{t}=q_{m}+q_{g}+q_{w} \\
q_{t} f_{g}=q_{g}+q_{m} f_{g} \\
h=h_{g}+h_{m}+h_{w}
\end{gathered}
$$

Also, at distance $\mathrm{X}$

$$
\left(q_{w}+q_{g}\right) f_{w} f_{g}=v w X
$$

or

$$
\left(q_{w}+q_{g}\right) f_{w} f_{g}=\Delta \rho g \cos (\theta) k_{v} f_{g}\left(\frac{k_{r w}}{\mu_{w}}\right) w X
$$

Of course, Equation 20 which actually represents a gas mass balance of the system may be replaced with water mass balance in the system as

$$
q_{t} f_{w}=q_{w}+q_{m} f_{w}
$$

Equations 16-22 include seven unknowns which can be solved for any $X \leq \mathrm{L}_{\mathrm{G}}$. Unknowns are $\mathrm{q}_{\mathrm{w}}, \mathrm{q}_{\mathrm{g}}$, $\mathrm{q}_{\mathrm{m}}, \mathrm{h}_{\mathrm{g}}, \mathrm{h}_{\mathrm{w}}, \mathrm{h}_{\mathrm{m}}$ and $\mathrm{d} \Phi$.

Table 1. Reservoir and fluid properties (based on Jenkins, 1984)

\begin{tabular}{ll}
\hline Property & Value \\
\hline Reservoir Length, $\mathbf{L}$ & $400 \mathrm{~m}$ \\
Reservoir Width, $\mathbf{w}$ & $400 \mathrm{~m}$ \\
Reservoir Height, $\mathbf{h}$ & $75 \mathrm{~m}$ \\
Horizontal Permeability, $\mathbf{k}_{\mathbf{h}}$ & $365.1604 \mathrm{E}-15 \mathrm{~m}^{2}(370.0 \mathrm{md})$ \\
Vertical Permeability, $\mathbf{k}_{\mathbf{v}}$ & $103.2318 \mathrm{E}-15 \mathrm{~m}^{2}(104.6 \mathrm{md})$ \\
Porosity, $\boldsymbol{\varphi}$ & 0.183 \\
Water Density, $\boldsymbol{\rho}_{\mathbf{w}}$ & $1000\left(\mathrm{~kg} / \mathrm{m}^{3}\right)$ \\
Gas Density, $\boldsymbol{\rho}_{\mathbf{g}}$ & $567\left(\mathrm{~kg} / \mathrm{m}^{3}\right)$ \\
Water Viscosity, $\boldsymbol{\mu}_{\mathbf{w}}$ & $0.3696 \mathrm{E}-03$ Pa.s $(0.3696 \mathrm{cp})$ \\
Gas Viscosity, $\boldsymbol{\mu}_{\mathbf{g}}$ & $0.0462 \mathrm{E}-03$ Pa.s $(0.0462 \mathrm{cp})$ \\
Dip Angle, $\boldsymbol{\theta}$ & $0^{\circ}, 15^{\circ}, 30^{\circ}, 45^{\circ}$ \\
\hline
\end{tabular}

Jenkins (1984) modified and simplified these non-linear equations to a closed-form solution. He also provided a good data set for defining a basic model. The modification seems not to be necessary in this paper as a MATLAB ${ }^{\circledR}$ program will be used which is capable of handling a set of non-linear equations but some of the data 
set generated by him will be used later (tables 1 and 2) to build a synthetic reservoir model. Also, Jenkins (1984) extended the above equations for estimating the increased recovery factor of WAG injection after waterflooding. Also important parameters which can maximize this recovery have been discussed in his work. The recovery equation will not be discussing here, nor being modified for a dipping model, but one might find it beneficial to modify and use such an equation together with the basic equations in this paper for having a rough estimate of recovery in a planned process.

Table 2. Saturation Distribution and Relative Mobility (based on Jenkins, 1984)

\begin{tabular}{cccc}
\hline \multirow{2}{*}{ Zone } & Water Saturation & $\frac{\mathbf{k}_{\mathbf{r g}}}{\boldsymbol{\mu}_{\mathbf{g}}},(1 / \mathrm{Pa} . \mathrm{s})$ & Water Relative Mobility, $\frac{\mathbf{k}_{\mathbf{r w}}}{\boldsymbol{\mu}_{\mathbf{w}}},(1 / \mathrm{Pa} . \mathrm{s})$ \\
\hline Gas & 0.4 & $8.719 \mathrm{E} 03$ & $0.006 \mathrm{E} 03$ \\
Mixed & 0.61 & $0.176 \mathrm{E} 03$ & $0.176 \mathrm{E} 03$ \\
Water & 0.66 & $0.018 \mathrm{E} 03$ & $0.276 \mathrm{E} 03$ \\
\hline
\end{tabular}

Using Equations 16-22, data provided in tables 1 and 2 and the MATLAB ${ }^{\circledR}$ program, we can compute the unknowns for these nonlinear equations. Besides the data available in these tables, we also assume that the total injection flow rate is equal to $0.007 \mathrm{~m}^{3} / \mathrm{s}$. Also, the WAG ratio in the mixed zone is equal to the water-gas mobility ratio which is 1 . So, the $f_{w}$ and $f_{g}$ are both equal to 0.5 .

Four different cases have been studied to accurately capture the effect of the dip angle on zone distribution in a WAG process. Figure 4 shows the boundaries for a horizontal model compared to a 15 degrees dipped model. The characteristics of the models (length, height, etc) are all similar. The dip angle, $\theta$, assumed to be positive in this case, which means a down-dip WAG injection is being applied to this model. As it is assumed that in models with different dip angle, microscopic sweep in gas segregated and WAG zones are constant (and also much higher than water segregated zone), the recovery is a direct function of volumetric sweep of gas and WAG zones, i.e. higher volumetric sweep of these two zones results in higher recovery. Even though both boundaries are close, it is still obvious that a dip angle extended the mixed zone, which means that higher recoveries can be achieved in this model. The role of a dip angle is more pronounced in Figures 5 and 6 where a horizontal model is compared to a 30 and 45 degrees dipped model. In these figures, complete segregation distance is increased considerably and also, a large region which was previously flooded by water in the horizontal model is now a part of mixed zone in non-horizontal model. Increased recovery for such a big region will increase average reservoir recovery, amazingly.

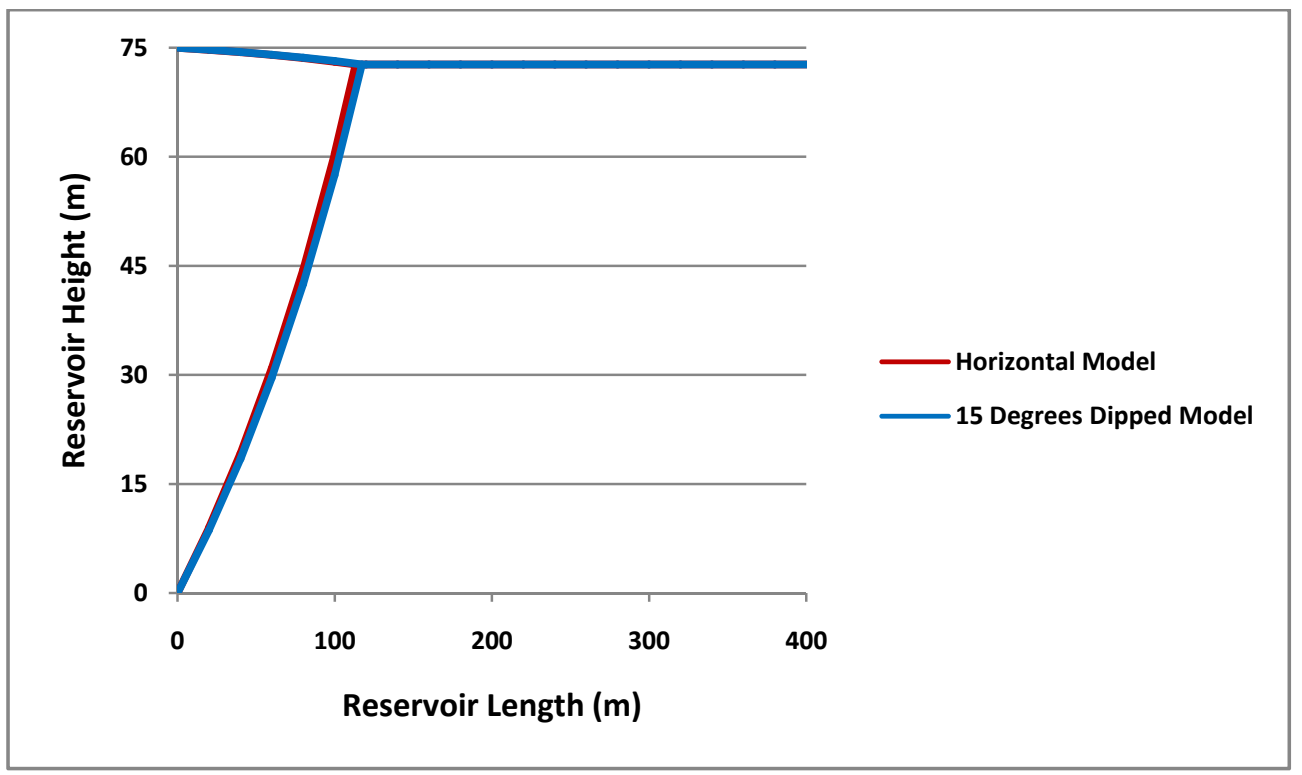

Figure 4. Zone distribution in WAG injection for horizontal and 15 degrees dipped reservoir models 


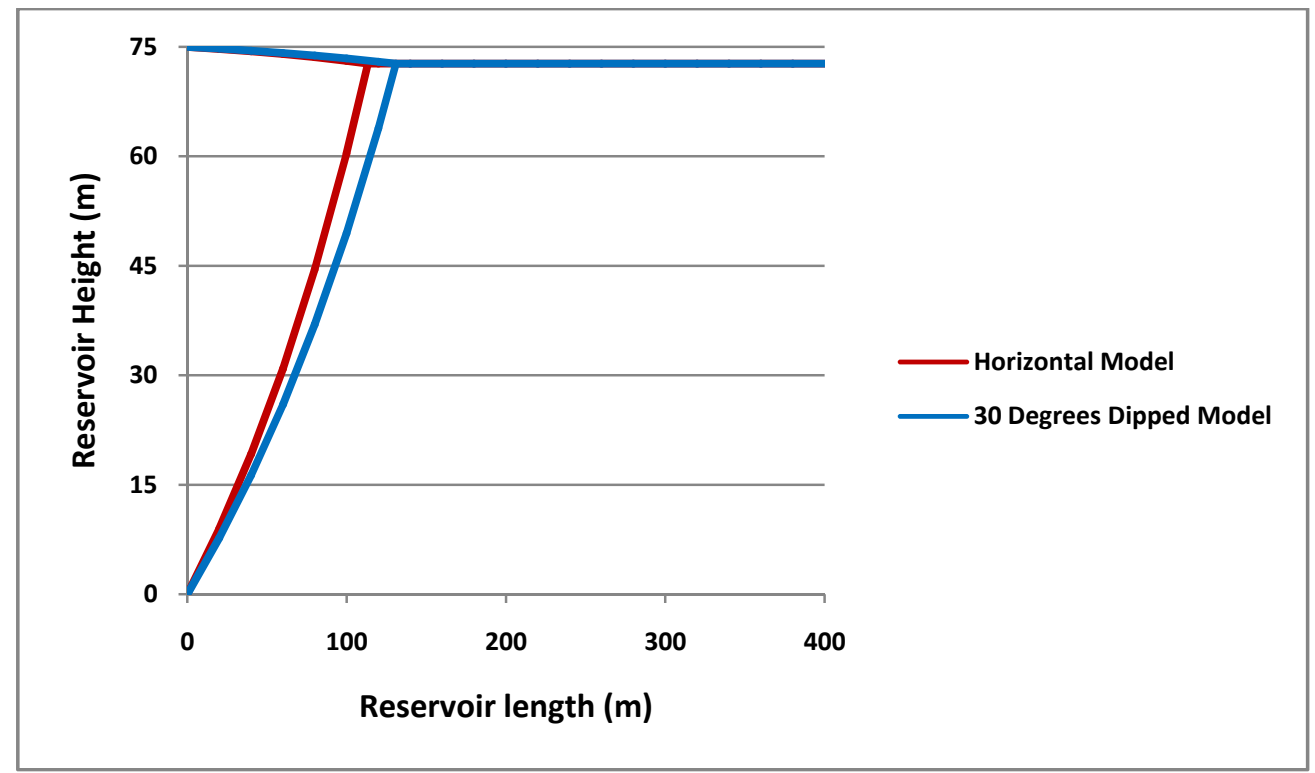

Figure 5. Zone distribution in WAG injection for horizontal and 30 degrees dipped reservoir models

As mentioned above, the discussion of WAG injection in a dipped reservoir assumed a down-dip WAG injection because of the positive sign of the dip angle, $\theta$. For an up-dip injection pattern $\theta$ will be negative, but $\cos (\theta)$ is still equal to $\cos (-\theta)$ in Equation 22b. So, if the flow potential, $d \Phi$, in the up-dip pattern is assumed to be the same as down-dip pattern, the zonal boundaries will be the same and the advantages of both cases are equal. But practically, we know that for a specific reservoir, the injectivity is limited. So, in the up-dip case the hydraulic head can help to have a higher flow potential compared to down-dip pattern. This increased flow potential will result in additional total flow rate and viscous force which can extend mixed zone in an up-dip model.

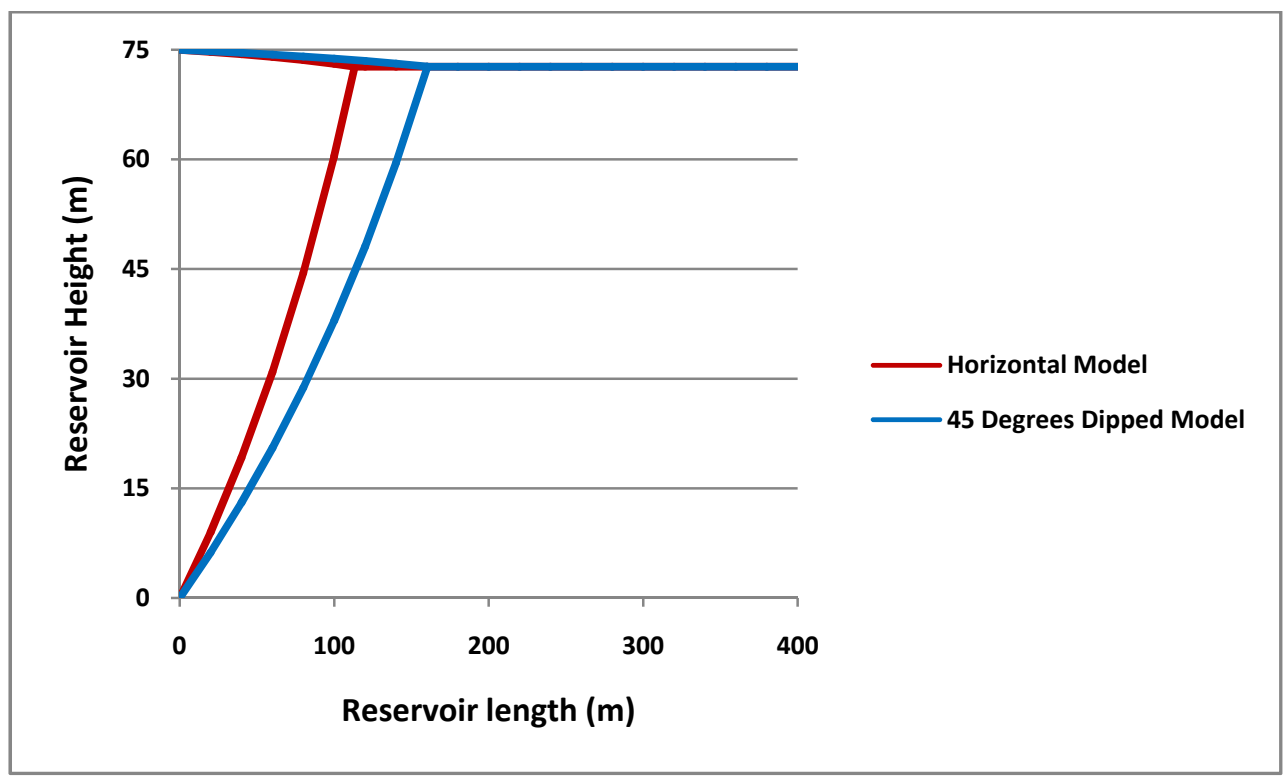

Figure 6. Zone distribution in WAG injection for horizontal and 45 degrees dipped reservoir models

\section{Conclusion}

An analytical equation has been derived that can be helpful for designing and analyzing the macroscopic sweep behavior of a reservoir-scale WAG process in dipped fields, especially when there are limited possibilities for simulation studies.

A comparison of horizontal and dipped reservoirs based on this mathematical derivation shows that an 
application of WAG injection in dipped models can be even more advantageous due to the extension of mixed zone. Such an extension of the mixed zone in a dipped reservoir mostly happens in the region that is supposed to be waterflooded in a horizontal reservoir, and not gasflooded, which makes it another favorable point for increased recovery. This increase takes place due to the fact that oil recovery in the mixed zone is considerably higher than in the waterflooded zone, and quite close to or even higher than in the gas zone.

Finally, assuming that the flow potential in up-dip and down-dip WAG injection cases are similar, the zonal boundaries will be the same. But it is obvious that because of the hydraulic head the injectivity can be somewhat different in these cases. Having the modified flow potential in each up-dip or down-dip case, it is possible to anticipate the zonal boundaries, and especially mixed zone borders.

\section{Acknowledgements}

Authors are sincerely grateful to Odd Steve Hustad, Torleif Holt and Masoud Asadollahi for their valuable comments and helps.

\section{References}

Blackwell, R. J., Terry, W. M., Rayne, J. R., Lindley, D. C., \& Henderson, J. R. (1960). Recovery of Oil by Displacements with Water-Solvent Mixtures. PETROLEUM TRANSACTIONS, AIME, 219, 293-300.

Buckley, S. E., \& Leverett, M. C. (1941). Mechanism of Fluid Displacement in Sands. Trans., AIME, 146, 107.

Caudle, B. H., \& Dyes, A. B. (1958). Improving Miscible Displacement by Gas-Water Injection. PETROLEUM TRANSACTIONS, AIME, 213, 281-284.

Dyes, A. B., Caudle, B. H., \& Erickson, R. A. (1954). Oil Production After Breakthrough as Influenced by Mobility Ratio. PETROLEUM TRANSACTIONS, AIME, 6(4), 27-32. http://dx.doi.org/10.2118/309-G

Faisal, A., Bisdom, K., Zhumabek, B., Mojaddam Zadeh, A., \& Rossen, W. R. (2009). Injectivity and Gravity Segregation in WAG and SWAG Enhanced Oil Recovery. SPE 124197 presented at the 2009 SPE Annual Technical Conference and Exhibition held in New Orleans, Louisiana, USA. http://dx.doi.org/10.2118/124197-MS

Jamshidnezhad, M., Shen, C., Kool, P., Mojaddam Zadehh, A., \& Rossen, W. R. (2010). Improving Injectivity To Fight Gravity Segregation in Gas Enhanced Oil Recovery. SPE Journal, 15(1), 91-104. http://dx.doi.org/10.2118/112375-PA

Jenkins, M. K. (1984). An Analytical Model for Water/Gas Miscible Displacements. SPE/DOE paper 12632 presented at the SPE/DOE Fourth Symposium on Enhanced Oil Recovery, Tulsa, OK. http://dx.doi.org/10.2118/12632-MS

Lake, L. W. (1989). Enhanced Oil Recovery. Englewood Cliffs, NJ: Prentice Hall.

MATLAB®. (2012). MathWorks ${ }^{\circledR}$. Retrieved February 9, 2012, from www.mathworks.com

Pande, K. K. (1992). Effects of Gravity and Viscous Crossflow on Hydrocarbon Miscible Flood Performance in Heterogeneous Reservoirs. SPE 24935 presented at the 67th Annual Technical Conference of the Society of Petroleum Engineers held in Washington, DC. http://dx.doi.org/10.2118/24935-MS

Rossen, W. R., van Duijn, C. J., Nguyen, Q. P., Shen, C., \& Vikingstad, A. K. (2010). Injection Strategies To Overcome Gravity Segregation in Simultaneous Gas and Water Injection Into Homogeneous Reservoirs. SPE Journal, 15(1), 76-90. http://dx.doi.org/10.2118/99794-PA

Sohrabi, M., Danesh, A., Tehrani, D. H., \& Jamiolahmady, M. (2008). Microscopic Mechanism of Oil Recovery By Near-Miscible Gas Injection. TRANSPORT IN POROUS MEDIA, 72(3), 351-367. http://dx.doi.org/10.1007/s11242-007-9154-z

Stone, H. L. (1982). Vertical Conformance in an Alternating Water-Miscible Gas Flood. SPE 11130 presented at 57th Annual Fall Technical Conference and Exhibition of the Society of Petroleum Engineers of AIME, New Orleans, LA. http://dx.doi.org/10.2118/11130-MS

Thomas, F. B., Holowach, N., Zhou, X. L., Bennion, D. B., \& Bennion, D. W. (1994). Miscible or Near-Miscible Gas Injection, Which Is Better? SPE/DOE 27811 presented at the SPE/DOE Ninth Symposium on Improved Oil Recovery, Tulsa, Oklahoma. http://dx.doi.org/10.2118/27811-MS 